\title{
Pneumonia associada a ventilação mecânica e cuidados preventivos de enfermagem:
}

\section{revisão de literatura}

\section{Pneumonia associated with mechanical ventilation and preventive nursing care: literature review \\ Neumonía asociada a ventilación mecánica y cuidados preventivos de enfermería: revisión de la}

\section{literatura}

Lisianne Natália Santos Silva

ORCID: https://orcid.org/0000-0002-2574-399X Centro Universitário de Ciências e Tecnologia do Maranhão, Brasil E-mail: lisyanenathalia6@gmail.com

Laianny Luize Lima e Silva

ORCID: https://orcd.org/.0000-0003-2553-6656

Universidade Federal do Piauí, Brasil

E-mail: laiannyluizelimaesilva@gmail.com

Wenderson Costa da Silva

ORCID: https://orcid.org/0000-0001-6031-9775

Universidade Estadual do Maranhão, Brasil

E-mail: wendersoncosta09@hotmail.com

Letícia Aparecida Santos Silva

ORCID: https://orcid.org/0000-0002-8000-7071

Centro Universitário de Ciências e Tecnologia do Maranhão, Brasil E-mail: benjamym60@gmail.com

Brunna Matos Sousa

ORCID: https://orcid.org/0000-0002-7009-6488 Centro Universitário de Ciências e Tecnologia do Maranhão, Brasil E-mail: brunnamattos4@gmail.com

Marconny Lira da Silva

ORCID: https://orcid.org/0000-0003-0334-9294

Faculdade Integrada Norte do Paraná, Brasil

E-mail: marconnylirads@hotmail.com

Hádila Giovanna Santos Siqueira Cunha

ORCID: https://orcid.org/0000-0002-8401-6119

Universidade Federal do Maranhão, Brasil E-mail: hadilagiovanna@hotmail.com

Márcia Sousa Santos

ORCID: https://orcid.org/0000-0002-6517-0479

Centro Universitário de Ciências e Tecnologia do Maranhão, Brasil

E-mail: mssenfermeira@gmail.com

Hálmisson D'Árley Santos Siqueira

ORCID: https://orcid.org/0000-0001-9831-5892

Centro Universitário de Ciências e Tecnologia do Maranhão, Brasil

E-mail: halmisson@yahoo.com.br

Francisléia Falcão França Santos Siqueira

ORCID: https://orcid.org/0000-0001-8783-5139

Universidade Estadual do Maranhão, Brasil

E-mail: gpmsaude@gmail.com

Maria Alice Souza Vitorio

ORCID: https://orcid.org/0000-0003-0407-6099

Centro Universitário de Ciências e Tecnologia do Maranhão, Brasil E-mail: Souzamaria2823@gmail.com

Ana Paula Cunha Duarte

ORCID: https://orcid.org/0000-0002-2898-5388

Universidade Estadual do Maranhão, Brasil

E-mail: anapduarte002@gmail.com

Jaqueline Brito da Costa

ORCID: https://orcid.org/0000-0002-2570-1714

Centro Universitário de Ciências e Tecnologia do Maranhão, Brasil

E-mail: Jaquelinebritto2012@gmail.com

Paulo Vicente Dourado

ORCID: https://orcid.org/0000-0002-0101-3423

Faculdade Malta, Brasil

E-mail: Paulo.vdourado@yahoo.com.br 


\author{
Juliana Helen Almeida de Lima \\ ORCID: https://orcid.org/0000-0002-1867-6450 \\ Faculdade Maurício de Nassau, Brasil \\ E-mail: jhelenlima22@gmail.com \\ Jairina Nunes Chaves \\ ORCID: https://orcid.org/0000-0002-3547-6901 \\ Universidade Estadual do Maranhão, Brasil \\ E-mail: inanunes@hotmail.com \\ Helvecyane Oliveira Barros \\ ORCID: https://orcid.org/0000-0002-5261-9249 \\ Centro Universitário Maurício de Nassau, Brasil \\ E-mail: vecyanebarros018@gmail.com \\ Samanta Rodrigues das Neves \\ ORCID: https://orcid.org/0000-0002-8280-0639 \\ Faculdade de Ciências Humanas de Curvelo, Brasil \\ E-mail: samantarodriguesdasneves404@gmail.com \\ Juliana de Sousa Oliveira Ximenes Cruz \\ ORCID: https://orcid.org/0000-0003-3430-171X \\ Centro Universitário de Ciências e Tecnologia do Maranhão, Brasil \\ E-mail: xjuliana865@gmail.com
}

\begin{abstract}
Resumo
A pneumonia associada à ventilação (PAV) é definida como uma infecção pulmonar que surge entre 48 a 72 horas após a intubação endotraqueal e início da ventilação mecânica invasiva (VMI), sendo uma das complicações mais temíveis da terapia intensiva. O estudo tem como objetivo geral analisar as ações de enfermagem na prevenção da pneumonia associada a ventilação mecânica. Trata-se de uma revisão integrativa da literatura, descritiva e exploratória, na qual utilizou-se a seguinte questão norteadora "Quais ações de enfermagem contribuem na prevenção da pneumonia associada a ventilação mecânica?", aplicada a estratégia PICO e com uso do booleano "AND" nas bases Bireme, Cinahl e Pubmed via Medline. Ao todo foram selecionados 12 artigos conforme os critérios previamente estabelecidos, a maioria dos estudos abordou sobre o nível de conhecimento dos profissionais de enfermagem quanto as medidas de prevenção em pneumonia associada a ventilação mecânica, além da adesão dos profissionais a ações preventivas e a utilização de estratégias que possam ser aplicadas no dia-a-dia da prática clínica. Torna-se claro que a assistência prestada deve ser focada tanto no processo de adoecimento que o paciente vivencia quanto na prevenção de outras patologias, como é o caso da pneumonia, que pode gerar diversas complicações e até mesmo levar ao óbito.
\end{abstract}

Palavras-chave: Pneumonia associada à ventilação mecânica; Cuidados de enfermagem; Prevenção de doenças.

\begin{abstract}
Ventilator-associated pneumonia (VAP) is defined as a pulmonary infection that appears between 48 to 72 hours after endotracheal intubation and initiation of invasive mechanical ventilation (IMV), and is one of the most fearful complications of intensive care. The study's general objective is to analyze nursing actions in the prevention of ventilator-associated pneumonia. This is an integrative, descriptive and exploratory literature review, in which the following guiding question was used "Which nursing actions contribute to the prevention of ventilator-associated pneumonia?", applied to the PICO strategy and using Boolean "AND" in Bireme, Cinahl and Pubmed databases via Medline. Altogether 12 articles were selected according to the previously established criteria, most of the studies addressed the level of knowledge of nursing professionals regarding preventive measures in pneumonia associated with mechanical ventilation, in addition to the professionals' adherence to preventive actions and the use of strategies that can be applied in daily clinical practice. It is clear that the care provided must be focused both on the illness process that the patient experiences and on the prevention of other pathologies, such as pneumonia, which can generate several complications and even lead to death.
\end{abstract}

Keywords: Pneumonia, ventilator-associated; Nursing care; Prevention.

\title{
Resumen
}

La neumonía asociada al ventilador (NAV) se define como una infección pulmonar que aparece entre 48 a 72 horas después de la intubación endotraqueal y el inicio de la ventilación mecánica invasiva (VMI), y es una de las complicaciones más temibles de los cuidados intensivos. El objetivo general del estudio es analizar las acciones de enfermería en la prevención de la neumonía asociada al ventilador. Se trata de una revisión bibliográfica integradora, descriptiva y exploratoria, en la que se utilizó la siguiente pregunta orientadora "¿Qué acciones de enfermería contribuyen a la prevención de la neumonía asociada al ventilador?", Aplicada a la estrategia PICO y utilizando el booleano "AND" en Bireme, Cinahl. y bases de datos Pubmed a través de Medline. En total se seleccionaron 12 artículos de acuerdo con los criterios previamente establecidos, la mayoría de los estudios abordaron el nivel de conocimiento de los profesionales de enfermería sobre las medidas preventivas en la neumonía asociada a la ventilación mecánica, además de la adherencia de los profesionales a las acciones preventivas y el uso de estrategias que se puede aplicar en la práctica clínica diaria. Es claro que la atención brindada debe estar enfocada tanto en el 
proceso de enfermedad que vive el paciente como en la prevención de otras patologías, como la neumonía, que pueden generar diversas complicaciones e incluso llevar a la muerte.

Palabras clave: Neumonía asociada al ventilador; Cuidado de enfermera; Prevención.

\section{Introdução}

A pneumonia associada à ventilação (PAV) é definida como uma infecção pulmonar que surge entre 48 a 72 horas após a intubação endotraqueal e início da ventilação mecânica invasiva (VMI), como também até 48 horas após a retirada do tubo ou extubação, sendo uma das complicações mais temíveis da terapia intensiva (Silva \& Moura, 2016; Almeida et al., 2015)

É observado que PAV é uma das maiores causas de infecções nosocomiais, que são doenças contraídas ou suspeitas na função de estadia em algum estabelecimento de saúde, levando ao aumento das taxas de infecções, aumento do tempo de internação e ocasionalmente aumento dos índices de mortalidade. Esse tipo de complicação se torna bem difícil devido à falta de um padrão específico de diagnóstico, nem todos os critérios de febre, presença de secreção purulenta, ou leucocitose pode estar relacionada a doença, porém outros padrões podem ser considerados, tais como hipoxemia, confusão mental, sepse ou choque. A biópsia do pulmão também pode ser utilizada para um diagnóstico definitivo, no entanto, este procedimento não é rotineiramente realizado (Ribeiro et al., 2016).

Entre as medidas de prevenção destinadas para a assistência de enfermagem ao paciente crítico com PAV, pode-se citar: a higienização das mãos que é um método individual e simples, sendo realizada antes e depois de qualquer procedimento, para que possa minimizar a migração de microrganismo para outros sítios; a higienização bucal dos pacientes em terapia intensiva, importante para reduzir a proliferação de bactérias, além da aspiração de secreções quando necessárias (Gonçalves et al., 2016).

Outra estratégia que tem sido utilizada refere-se à criação de bundles de cuidados, onde reúne algumas intervenções que são baseadas em evidências e quando realizadas adequadamente, alcançam uma melhoria na assistência em saúde (Almeida et al., 2015).

Desta forma, o estudo tem como objetivo geral analisar as ações de enfermagem na prevenção da pneumonia associada a ventilação mecânica, e especificamente identificar os fatores de risco para o desenvolvimento da pneumonia associada a ventilação mecânica dentro da UTI; descrever os fatores que influenciam na adesão de medidas de prevenção da pneumonia associada a ventilação mecânica e apontar bundles existentes para intervenção na prevenção da pneumonia associada a ventilação mecânica na UTI.

Entende-se que a pneumonia associada a ventilação mecânica é um grave problema de saúde pública, portanto tornase necessário adentrar-se a temática, e promover embasamento teórico aos profissionais de enfermagem, sobre medidas de cuidados prestados na terapia intensiva e como eles podem prevenir este tipo de complicação, servindo assim como um instrumento de prevenção e para debates futuros.

\section{Metodologia}

Trata-se de uma revisão integrativa da literatura, descritiva e exploratória, este delineamento de pesquisa foi escolhido por possibilitar a síntese de conteúdos publicados no meio científico sobre a temática "PNEUMONIA ASSOCIADA A VENTILAÇÃO MECÂNICA E CUIDADOS PREVENTIVOS DE ENFERMAGEM: revisão de literatura". Para nortear a estrutura e organização da revisão, o estudo utilizou a metodologia proposta por Alves e Oliveira (2016).

Segundo Sousa, Silva e Carvalho (2010) estudos de revisão integrativa demonstram ser importantes ferramentas para o campo da saúde, pois permite compilar diversas informações confiáveis para responder uma determinada questão específica e com ênfase na prática baseada em evidências, além disso permite combinar e analisar diferentes tipos de delineamento de 
pesquisa utilizando um rigor metodológico para avaliar todo o processo de análise dos estudos e reduzindo assim os riscos de vieses e erros.

A pesquisa descritiva caracterizar-se por analisar e registrar os fenômenos estudados, avaliando todos os detalhes desde a causa quanto os fatores que influenciam de forma direta ou indireta. Enquanto que a pesquisa exploratória reúne um agregado de informações delimitando o objeto de estudo, e as condições de manifestação do mesmo (Severino, 2017).

Utilizou-se a seguinte questão norteadora "Quais a ações de enfermagem contribuem na prevenção da pneumonia associada a ventilação mecânica?", com a pergunta definida fora aplicada a estratégia PICO, em que "P” indica a população ou objeto de interesse no estudo, "I" a intervenção, "C" o comparador e "O" desfecho ou resultados, conforme o Quadro 1.

Quadro 1. Componentes da estratégia PICO e descritores definidos. Brasil, 2021.

\begin{tabular}{|c|c|c|c|c|}
\hline \multicolumn{2}{|c|}{ ELEMENTOS } & DECS & MESH TERMS & TÍTULO CINAHL \\
\hline $\mathbf{P}$ & $\begin{array}{c}\text { Pneumonia } \\
\text { associada a } \\
\text { ventilação } \\
\text { mecânica }\end{array}$ & $\begin{array}{l}\text { "Pneumonia Associada à } \\
\text { Ventilação Mecânica" } \\
\text { "Pneumonia, Ventilator- } \\
\text { Associated" } \\
\text { "Neumonía Asociada al } \\
\text { Ventilador" }\end{array}$ & $\begin{array}{c}\text { "Pneumonia, Ventilator- } \\
\text { Associated" }\end{array}$ & $\begin{array}{c}\text { "Pneumonia, Ventilator- } \\
\text { Associated" }\end{array}$ \\
\hline I & $\begin{array}{c}\text { Ações de } \\
\text { enfermagem }\end{array}$ & $\begin{array}{c}\text { “Cuidados de Enfermagem” } \\
\text { "Nursing Care” } \\
\text { "Atención de Enfermería" }\end{array}$ & "Nursing Care" & "Nursing Care" \\
\hline $\mathbf{C}$ & - & - & - & - \\
\hline $\mathbf{O}$ & Prevenção & $\begin{array}{c}\text { "Prevenção de Doenças" } \\
\text { "Disease Prevention" } \\
\text { "Prevención de Enfermedades", }\end{array}$ & "Prevention" & "Prevention" \\
\hline
\end{tabular}

Fonte: Autores.

A pergunta foi estruturada e classificada conforme os elementos da estratégia PICO, não foi determinado o elemento comparador pois não era um item componente da pergunta estabelecida, e posteriormente cada termo foi definido por meio das buscas realizadas nos descritores de Ciências da Saúde (Decs), Medical Subject Headings (Mesh terms), e o título Cinahl.

Posteriormente esses termos foram combinados para serem aplicados nas bases definidas sendo elas Centro LatinoAmericano e do Caribe de Informação em Ciências da Saúde (Bireme), Medical Literature Analysis and Retrieval System Online (Pubmed via Medline) e Cumulative Index to Nursing and Allied Health Literature (Cinahl), como pode ser observado no Quadro 2.

Quadro 2. Estratégias de busca utilizadas nas bases de dados BIREME, PUBMED e CINAHL. Brasil, 2021.

\begin{tabular}{|c|l|c|c|c|}
\hline $\begin{array}{c}\text { BASE DE } \\
\text { DADOS }\end{array}$ & \multicolumn{1}{|c|}{ ESTRATÉGIA DE BUSCA } & RESULTADOS & SELECIONADOS \\
\hline Bireme & $\begin{array}{l}\text { (Pneumonia, Ventilator-Associated) } \\
\text { AND (Nursing Care) AND (Disease } \\
\text { Prevention) }\end{array}$ & 95 & 31 \\
\hline Cinahl & $\begin{array}{l}\text { pneumonia, ventilator-associated } \\
\text { AND nursing care AND prevention }\end{array}$ & 387 & 27 & 04 \\
\hline PubMed & $\begin{array}{l}(\text { Pneumonia, Ventilator-Associated) } \\
\text { AND (Nursing Care) AND } \\
\text { (prevention and control) }\end{array}$ & 369 & 39 & 03 \\
\hline
\end{tabular}


Com os descritores definidos, estes foram combinados em cada base de dados, gerando endereços de busca em cada uma delas e resultado em números de artigos diversos em cada base, que foram filtrados e selecionados posteriormente conforme os seguintes critérios: estudos completos publicados em periódicos, que fossem artigos científicos, publicados nos últimos seis anos, sendo excluídos relatos de casos, dissertações, estudos de casos e capítulos de livros.

A análise dos estudos foi feita de forma descritiva e com cálculos matemáticos em que inicialmente foi feita a filtragem dos estudos, seguida da seleção prévia dos estudos por meio de títulos e resumos, e logo após a leitura dos textos completos, definir os estudos que fariam parte do quadro de resultados. Na qual foram categorizados e organizados em quadros e tabelas para facilitar a compreensão das etapas realizadas em relação aos dos dados obtidos, além das informações mais pertinentes em relação a temática.

\section{Resultados}

Ao todo foram selecionados 12 artigos conforme os critérios previamente estabelecidos. Todos os estudos apresentavam abordagem quantitativa (100\%). A maioria dos estudos estavam disponível no idioma inglês (83,3\%), em relação ao delineamento de estudo destacou-se os estudos transversais $(58,3 \%)$, tendo maior procedência da Jordânia $(33,3)$. As publicações dos estudos concentraram-se no ano de 2017 (25\%), com nível de evidência de 2c (66,6\%) e grau de recomendação B $(41,6 \%)$. Os critérios de avaliação de elegibilidade dos estudos foram feitos com base a classificação proposta Oxford Center for Evidence-Based Medicine (2009), conforme a Tabela 1. 
Tabela 1. Análise descritiva das produções científicas acerca da temática "PNEUMONIA ASSOCIADA A VENTILAÇÃO MECÂNICA: cuidados preventivos de enfermagem”. Brasil, 2021. (N=12)

\begin{tabular}{|c|c|c|}
\hline VARIÁVEIS & $\mathbf{N}$ & $\%$ \\
\hline \multicolumn{3}{|l|}{ Base de dados } \\
\hline Bireme & 05 & $41,6 \%$ \\
\hline Cinahl & 04 & $33,3 \%$ \\
\hline PubMed & 03 & $25 \%$ \\
\hline \multicolumn{3}{|l|}{ Delineamento de pesquisa } \\
\hline Caso-controle & 01 & $8,3 \%$ \\
\hline Experimental & 01 & $8,3 \%$ \\
\hline Quase-experimental & 02 & $16,6 \%$ \\
\hline Transversal & 08 & $66,6 \%$ \\
\hline \multicolumn{3}{|l|}{ Abordagem do estudo } \\
\hline Quantitativo & 12 & $100 \%$ \\
\hline \multicolumn{3}{|l|}{ Ano } \\
\hline 2016 & 02 & $16,6 \%$ \\
\hline 2017 & 03 & $25 \%$ \\
\hline 2018 & 02 & $16,6 \%$ \\
\hline 2019 & 01 & $8,3 \%$ \\
\hline 2020 & 02 & $16,6 \%$ \\
\hline 2021 & 02 & $16,6 \%$ \\
\hline \multicolumn{3}{|l|}{ País } \\
\hline Arábia Saudita & 01 & $8,3 \%$ \\
\hline Brasil & 02 & $16,6 \%$ \\
\hline China & 01 & $8,3 \%$ \\
\hline Espanha & 01 & $8,3 \%$ \\
\hline Índia & 01 & $8,3 \%$ \\
\hline Jordânia & 04 & $33,3 \%$ \\
\hline Turquia & 02 & $16,6 \%$ \\
\hline \multicolumn{3}{|l|}{ Idioma } \\
\hline Inglês & 10 & $83,3 \%$ \\
\hline Português & 02 & $16,2 \%$ \\
\hline \multicolumn{3}{|l|}{ Nível de evidência } \\
\hline $1 \mathrm{c}$ & 03 & $25 \%$ \\
\hline $2 \mathrm{c}$ & 08 & $66,6 \%$ \\
\hline $3 b$ & 01 & $8,3 \%$ \\
\hline \multicolumn{3}{|l|}{ Grau de recomendação } \\
\hline A & 03 & $25 \%$ \\
\hline $\mathrm{B}$ & 05 & $41,6 \%$ \\
\hline $\mathrm{C}$ & 04 & $33,3 \%$ \\
\hline
\end{tabular}

Legenda: N = número; $\%$ = percentual. Fonte: Dados da pesquisa, 2021.

No geral os estudos apresentaram um bom nível de evidência e informações detalhadas para a síntese de conteúdo, e estes também foram organizados conforme os critérios de inclusão previamente estabelecido para auxiliar na identificação e características pormenores dos estudos, como demonstrado no Quadro 3. 
Research, Society and Development, v. 11, n. 3, e19611326022, 2022

(CC BY 4.0) | ISSN 2525-3409 | DOI: http://dx.doi.org/10.33448/rsd-v11i3.26022

Quadro 3. Publicações incluídas conforme o título do artigo, autor, ano, objetivo principal, delineamento e perfil amostral. Caxias, MA, Brasil. 2021. (N=12)

\begin{tabular}{|c|c|c|c|c|}
\hline Título do artigo & Autor/ ano & Objetivo principal & Delineamento & Perfil amostral \\
\hline $\begin{array}{l}\text { Adesão ao protocolo de pneumonia } \\
\text { associado à ventilação mecânica }\end{array}$ & $\begin{array}{l}\text { Zigart et al. } \\
\text { (2019) }\end{array}$ & $\begin{array}{l}\text { Conhecer a adesão da equipe de enfermagem } \\
\text { ao protocolo de pneumonia associada à } \\
\text { ventilação mecânica nas Unidades de } \\
\text { Terapia Intensiva. }\end{array}$ & Transversal & $\begin{array}{l}\text { Foram feitas } 945 \text { checagens de pacientes em ventilação } \\
\text { mecânica, sendo } 538(56,93 \%) \text { do sexo masculino, com a } \\
\text { faixa etária de maior prevalência de } 61 \text { a } 80 \text { anos }(n=427 \text {; } \\
45,19 \%) \text { e a menos prevalente de até } 20 \text { anos }(n=19 ; \\
2,01 \%) \text {; }\end{array}$ \\
\hline $\begin{array}{l}\text { Prevention of ventilator-associated } \\
\text { pneumonia: Use of the care bundle } \\
\text { approach }\end{array}$ & $\begin{array}{l}\text { Alcan, } \\
\text { Korkmaz e } \\
\text { Uyar }(2016)\end{array}$ & $\begin{array}{l}\text { Investigar o efeito do uso de bundles de } \\
\text { cuidados com a inclusão de higiene das } \\
\text { mãos e monitoramento da pressão do } \\
\text { balonete do tubo endotraqueal na VAP. }\end{array}$ & $\begin{array}{l}\text { Quase- } \\
\text { experimental }\end{array}$ & $\begin{array}{l}\text { Foram incluídos no estudo. Um total de } 222 \text { pacientes } \\
\text { foram admitidos na UTI. Cento e vinte e oito desses } \\
\text { pacientes preencheram os critérios da pesquisa em } \\
\text { inclusão. }\end{array}$ \\
\hline $\begin{array}{l}\text { Evidence-Based Practices for } \\
\text { Preventing Ventilator-Associated } \\
\text { Pneumonia in Intensive Care Nursing: } \\
\text { Knowledge and Practice }\end{array}$ & $\begin{array}{l}\text { Aysegul, } \\
\text { Oznur e Asiye } \\
(2020)\end{array}$ & $\begin{array}{l}\text { Determinar o conhecimento dos enfermeiros } \\
\text { de UTI e a implementação de métodos } \\
\text { baseados em evidências práticas para a } \\
\text { prevenção de PAV. }\end{array}$ & Transversal & $\begin{array}{l}\text { O estudo incluiu } 102 \text { enfermeiras com pelo menos seis } \\
\text { meses ou mais de experiência em UTI e em pacientes } \\
\text { críticos. }\end{array}$ \\
\hline $\begin{array}{l}\text { Compliance of Nurses and Hospitals } \\
\text { With Ventilator Associated } \\
\text { Pneumonia Prevention Guidelines A } \\
\text { Middle Eastern Survey }\end{array}$ & $\begin{array}{c}\text { Aloush et al. } \\
\text { (2018) }\end{array}$ & $\begin{array}{l}\text { Avaliar a conhecimento de enfermeiras em } \\
\text { hospitais com VAP e do uso de diretrizes de } \\
\text { prevenção, os fatores que afetam seu nível } \\
\text { de conformidade e as barreiras. }\end{array}$ & Transversal & $\begin{array}{l}\text { Os participantes eram de } 17 \text { centros médicos em } 3 \text { países: } \\
\text { Jordânia ( } 185 \text { participantes), Arábia Saudita ( } 88) \text {, e } \\
\text { Egito (200). O estudo foi concedido ético permissão das } \\
\text { universidades dos pesquisadores e os hospitais } \\
\text { participantes. }\end{array}$ \\
\hline $\begin{array}{l}\text { Nurses' implementation of ventilator- } \\
\text { associated pneumonia prevention } \\
\text { guidelines: an observational study in } \\
\text { Jordan }\end{array}$ & Aloush (2018) & $\begin{array}{l}\text { Avaliar a conformidade dos enfermeiros } \\
\text { com as diretrizes de prevenção de } \\
\text { pneumonia associada à ventilação mecânica } \\
\text { e os fatores que influenciam sua } \\
\text { conformidade. }\end{array}$ & Transversal & $\begin{array}{l}\text { Um total de } 130 \text { enfermeiras, dos quais apenas } 100 \\
\text { concordaram em participar, dando uma taxa de resposta de } \\
77 \% \text {. }\end{array}$ \\
\hline $\begin{array}{l}\text { Evidence-based guidelines for } \\
\text { prevention of ventilator-associated } \\
\text { pneumonia: Evaluation of intensive } \\
\text { care unit nurses' adherence }\end{array}$ & $\begin{array}{l}\text { Darawad, } \\
\text { Sa'Aleek e } \\
\text { Shawashi } \\
\text { (2017) }\end{array}$ & $\begin{array}{l}\text { Avaliar o nível de adesão das enfermeiras } \\
\text { jordanianas às diretrizes de VAP. }\end{array}$ & Transversal & $\begin{array}{l}\text { Três hospitais de médio e grande porte que foram } \\
\text { selecionados aleatoriamente de hospitais que representam } \\
\text { diferentes setores de saúde na região central da Jordânia. }\end{array}$ \\
\hline $\begin{array}{l}\text { Knowledge level of nurses in Jordan } \\
\text { on ventilator-associated pneumonia } \\
\text { and preventive measures }\end{array}$ & $\begin{array}{l}\text { Hassan e } \\
\text { Wahsheh } \\
(2016)\end{array}$ & $\begin{array}{l}\text { Identifique o nível dos enfermeiros } \\
\text { conhecimento sobre pneumonia associada à } \\
\text { ventilação e prevenção após um programa } \\
\text { educacional e identificar as razões para não } \\
\text { aplicar medidas de prevenção da pneumonia } \\
\text { associada à ventilação entre enfermeiras na } \\
\text { Jordânia. }\end{array}$ & Transversal & $\begin{array}{l}\text { Questionário autorreferido de } 428 \text { enfermeiros que } \\
\text { trabalhavam em unidades de terapia intensiva. }\end{array}$ \\
\hline
\end{tabular}


Research, Society and Development, v. 11, n. 3, e19611326022, 2022

(CC BY 4.0) | ISSN 2525-3409 | DOI: http://dx.doi.org/10.33448/rsd-v11i3.26022

\begin{tabular}{|c|c|c|c|c|}
\hline $\begin{array}{l}\text { Nursing workload and compliance } \\
\text { with non-pharmacological measures } \\
\text { to prevent ventilator-associated } \\
\text { pneumonia: a multicentre study }\end{array}$ & $\begin{array}{l}\text { Jam et al. } \\
(2018)\end{array}$ & $\begin{array}{l}\text { Avaliar o impacto da carga de trabalho de } \\
\text { enfermagem na adesão às medidas não } \\
\text { farmacológicas de prevenção da pneumonia } \\
\text { associada à ventilação mecânica. }\end{array}$ & Transversal & $\begin{array}{l}\text { Um total de } 97 \text { enfermeiros foram estudados; } 76(79 \%) \\
\text { foram observados. Houve } 327 \text { observações (média de } 4 \cdot 30 \\
\text { observações por enfermeira). O questionário mostrou uma } \\
\text { boa linha de base conhecimento das medidas preventivas. }\end{array}$ \\
\hline $\begin{array}{l}\text { The clinical value of comprehensive } \\
\text { nursing intervention in preventing } \\
\text { ventilator-associated pneumonia }\end{array}$ & $\begin{array}{l}\text { Fan, Chu, } \\
\text { Jiang e Du } \\
\text { (2021) }\end{array}$ & $\begin{array}{l}\text { Avaliar o valor clínico da intervenção } \\
\text { abrangente de enfermagem na prevenção da } \\
\text { pneumonia associada à ventilação mecânica } \\
\text { (VAP). }\end{array}$ & Caso-controle & $\begin{array}{l}116 \text { pacientes que se apresentam ao nosso hospital de } \\
\text { junho de } 2018 \text { a dezembro de } 2019 \text { para ventilação } \\
\text { mecânica participaram do estudo atual após obter } \\
\text { consentimento informado por escrito e aprovação do } \\
\text { comitê de ética do hospital. }\end{array}$ \\
\hline $\begin{array}{l}\text { Educação para prevenção da } \\
\text { pneumonia associada à ventilação } \\
\text { mecânica em unidade de terapia } \\
\text { intensiva }\end{array}$ & $\begin{array}{l}\text { Branco et al. } \\
\quad(2020)\end{array}$ & $\begin{array}{l}\text { Avaliar a adesão da enfermagem ao bundle } \\
\text { de prevenção à Pneumonia Associada à } \\
\text { Ventilação Mecânica e a taxa de incidência, } \\
\text { antes e após Educação Permanente. }\end{array}$ & $\begin{array}{c}\text { Quase- } \\
\text { experimental }\end{array}$ & $\begin{array}{l}\text { Estudo realizado com } 302 \text { pacientes em ventilação } \\
\text { mecânica internados em Unidade de Terapia Intensiva. }\end{array}$ \\
\hline $\begin{array}{l}\text { Critical care nurses' compliance and } \\
\text { barriers toward ventilatorassociated } \\
\text { pneumonia prevention guidelines: } \\
\text { cross-sectional survey }\end{array}$ & $\begin{array}{l}\text { Al-Sayaghi } \\
\text { (2021) }\end{array}$ & $\begin{array}{l}\text { Este estudo visa determinar a conformidade } \\
\text { de enfermeiras de cuidados intensivos com o } \\
\text { ventilador associado diretrizes de prevenção } \\
\text { de pneumonia e os fatores que afetar seu } \\
\text { cumprimento. }\end{array}$ & Transversal & $\begin{array}{l}\text { Um total de } 229 \text { convidados responderam ao questionário. } \\
\text { O escore médio de conformidade foi de } 85,9 \% \text {. Mais da } \\
\text { metade }(54 \%) \text { da amostra teve uma alta ou aceitável nível } \\
\text { de conformidade. }\end{array}$ \\
\hline $\begin{array}{l}\text { A Comparative Study to Assess the } \\
\text { Competency among Icu Nurses in } \\
\text { Using Ventilator Associated } \\
\text { Pneumonia Bundle to Prevent } \\
\text { Ventilator Associated Pneumonia in } \\
\text { Selected Government and Private } \\
\text { Hospitals of New Delhi. }\end{array}$ & $\begin{array}{c}\text { Shahnaz, } \\
\text { Bhadwaj e } \\
\text { Tamag (2018) }\end{array}$ & $\begin{array}{l}\text { Avaliar e comparar o nível de competência } \\
\text { entre enfermeiras de UTIs governamentais e } \\
\text { privadas hospitais no uso do Bundle VAP. }\end{array}$ & Experimental & $\begin{array}{l}30 \text { enfermeiras de UTI de hospital público e hospitais } \\
\text { privados. }\end{array}$ \\
\hline
\end{tabular}

Fonte: Base de dados (2021). 
A maioria dos estudos abordou sobre o nível de conhecimento dos profissionais de enfermagem quanto as medidas de prevenção em pneumonia associada a ventilação mecânica, além da adesão dos profissionais a ações preventivas e a utilização de estratégias que possam ser aplicadas no dia a dia da prática clínica.

Os bundles são cuidados aplicados de forma multidisciplinar e auditados pela Comissão de Controle de Infecção Hospitalar (CCIH), um método que não necessita de grandes tecnologias para sua realização, o que possibilita a aplicação em todas as UTI's e manejo dos profissionais sem sobrecarregar seus trabalhos (Almeida et al., 2015), conforme o Quadro 4.

Quadro 4 - Bundles de intervenções de enfermagem com orientações quanto a prevenção da pneumonia associada a ventilação mecânica. 2021.

\begin{tabular}{|c|l|}
\hline Autor/Ano & \multicolumn{1}{|c|}{ Intervenções } \\
\hline $\begin{array}{c}\text { Alcan, Korkmazv e Uyar (2016), Aloush } \\
\text { (2018), Aloush et al. (2018); Darawad, } \\
\text { Sa'aleek e Shawashi (2017) }\end{array}$ & Higiene das mãos antes e após contato com o paciente \\
\hline $\begin{array}{c}\text { Alcan, Korkmazv e Uyar (2016), Aloush } \\
\text { (2018), Aloush et al. (2018), Branco et al. } \\
\text { 2020, Darawad, Sa'aleek e Shawashi } \\
\text { (2017) }\end{array}$ & Higiene bucal com clorexidina \\
\hline $\begin{array}{c}\text { Aloush (2018), Aloush et al. (2018), } \\
\text { Darawad, Sa'aleek e Shawashi (2017), } \\
\text { Hassan e Wahsheh (2016) }\end{array}$ & Utilização de sistema fechado de aspiração \\
\hline $\begin{array}{c}\text { Alcan, Korkmazv e Uyar (2016), Aloush } \\
\text { (2018), Aloush et al. (2018), Darawad, } \\
\text { Sa'aleek e Shawashi (2017) }\end{array}$ & $\begin{array}{l}\text { Avaliação diária da prontidão dos pacientes para desmame do ventilador } \\
\text { mecânico }\end{array}$ \\
\hline \begin{tabular}{c} 
Branco et al. (2020), Zigart et al. (2019) \\
\hline
\end{tabular} & $\begin{array}{l}\text { Elevação da cabeceira do leito com angulação entre } 30^{\circ} \text { e } 45^{\circ} \text {, dependendo da } \\
\text { clínica do paciente }\end{array}$ \\
\hline
\end{tabular}

Fonte: Elaboração do autor.

A criação de bundles de cuidados, reúne algumas intervenções que são baseadas em evidências, quando realizadas alcançam uma melhoria na assistência em saúde. Os estudos demonstraram os resultados e eficácia das intervenções principalmente por meio da adesão dos profissionais enfermeiros a protocolos com medidas que tinham como foco evitar o desenvolvimento de pneumonia nos pacientes em ventilação mecânica.

\section{Discussão}

\section{Ações de enfermagem na prevenção da pneumonia associada a ventilação mecânica}

Entre as principais intervenções realizadas pela equipe de enfermagem com o intuito de prevenir a pneumonia associada a ventilação mecânica está a lavagem das mãos, uso de solução de clorexidina para higiene bucal, utilização de sistema fechado de aspiração e execução de diário de avaliação da prontidão dos pacientes para desmame do ventilador mecânico (Aloush et al., 2018; Darawad et al., 2017).

A pesquisa realizada por Aloush (2018) na Jordânia com 100 enfermeiros de diferentes hospitais demonstrou que nas UTI's onde os pacientes eram acompanhados por profissionais que realizavam avaliação diária da prontidão para o desmame, lavagem das mãos constantemente, utilizavam clorexidina para higienização bucal e sistema fechado de aspiração apresentavam uma menor taxa de pneumonia.

Corroborando com esse estudo, a pesquisa de Alcan, Korkmazv e Uyar (2016) feita na Turquia com 128 pacientes ao aplicar um pacote de intervenções que incluíam observação diária de prontidão para extubação, higiene das mãos e higiene 
bucal com clorexidina, mostrou que as taxas de pneumonia por ventilação mecânica reduziram de 15,9/10 dias de ventilação para 8,50/10 dias de ventilação.

De acordo com Hassan e Wahsheh (2016), o sistema fechado para os pacientes que estão em ventilação mecânica faz com que não necessite de desconexão do circuito para realizar manobras de sucção e limpeza, além de preservar a pressão positiva das vias aéreas.

Outra intervenção bastante relatada foi a elevação do leito entre $30^{\circ}$ e $45^{\circ}$, na qual Zigart et al. (2019) destaca que essa angulação promove a diminuição de reflexo e da aspiração de secreções nasofaríngeas, orofaríngeas e gastrointestinais, como também melhora os parâmetros ventilatórios e diminui o risco de pneumonia.

Neste sentido, a pesquisa feita em uma UTI de Porto Alegre verificou que após a aplicação de um pacote de intervenções preventivas aos pacientes em ventilação mecânica, combinando medidas executadas pela enfermagem, como cabeceira do leito levantada entre $30^{\circ}$ e $45^{\circ}$, dependendo da condição clínica do paciente; higiene oral usando clorexidina; posicionamento do filtro do ventilador acima da introdução do tubo endotraqueal, entre outras, mostrou que a densidade de incidência de pneumonia por ventilação mecânica diminuiu de 7,99 para 4,28 infecções/1000 ventiladores mecânicos dia (Branco et al., 2020).

Dessa forma, é perceptível que as intervenções estão sempre sendo aprimoradas, como mostra o estudo realizado na China com 116 pacientes que após comparar 2 grupos de enfermeiros, no qual um utilizava intervenções convencionais, como aspiração comum de expectoração, posicionamento do paciente, atendimento psicológico e conforto do ambiente, e o outro grupo com intervenções abrangentes, como enfermagem cognitiva e psicológica, posicionamento do paciente, umidade e temperatura adequada das vias aéreas, higiene oral e limpeza periódica dos tubos do ventilador, apontou que a incidência de pneumonia associada a ventilação mecânica foi inferior no grupo com intervenções abrangentes, apresentando também um menor tempo de hospitalização (Fan et al., 2021).

\section{Fatores de risco para o desenvolvimento da pneumonia associada à ventilação mecânica dentro da UTI}

No que se refere aos fatores de risco para o desenvolvimento de pneumonia associada à ventilação mecânica dentro da UTI, muitos agentes podem estar relacionados e variar de instituição para instituição. Neste aspecto, Fan et al. (2021) afirmam que, a enfermagem pode reduzir significativamente os fatores de risco, através de medidas tornem o ambiente e os procedimentos mais limpos e livres de microorganismos, e estimulem positivamente os pacientes. Ainda sobre a enfermagem, Hassan e Wahsheh (2016) sustentam a ideia de que o grau de conhecimento dos enfermeiros influência de forma positiva ou negativa o desenvolvimento de pneumonia.

Não há um consenso entre todos os estudiosos, com este intuito, pesquisas são feitas para melhor apresentar todos os fatores de risco ligados ao desenvolvimento da pneumonia associada a ventilação mecânica dentro da UTI. Em seu estudo AlSayaghi (2021), aponta como principais fatores de risco, alterações nos umidificadores e alterações nos circuitos dos aparelhos. Além disso, foram observadas barreiras na implementação de medidas de prevenção, o que consequentemente torna-se outro importante fator de risco.

Destaca-se ainda a quantidade de dias de internação hospitalar e a quantidade de dias em uso de ventilação mecânica (Branco et al., 2020). Ressalta-se também, a falta de realização da higienização oral dos pacientes em uso de ventilação mecânica e o não uso de clorexidina, como fatores relacionados à infecção (Aloush et al., 2018).

Por fim, é importante salientar que os fatores de risco não são isolados e agem em conjunto para ocasionar a infecção, podendo variar de acordo com a instituição, os profissionais e os pacientes (Al-Sayaghi, 2021). Neste ponto, é necessário haver padronização das medidas para prevenir infecções, porém, na prática, é observado que, muitos hospitais não apresentam 
Research, Society and Development, v. 11, n. 3, e19611326022, 2022

(CC BY 4.0) | ISSN 2525-3409 | DOI: http://dx.doi.org/10.33448/rsd-v11i3.26022

protocolos com medidas de prevenção, oferecendo muitos riscos aos pacientes (Darawad et al., 2017).

\section{Fatores que influenciam na adesão de medidas de prevenção da pneumonia associada a ventilação mecânica em UTI}

Muitas medidas de prevenção são criadas a fim de ajudar na recuperação dos pacientes e efetivar o cuidado, neste ponto, observa-se que diversos fatores podem influenciar a adesão dos profissionais a medidas de prevenção da pneumonia associada a ventilação mecânica, entre elas, destacam-se a falta de tempo dos profissionais para seguir os protocolos e a falta de conhecimento científico sobre os procedimentos realizados (Hassan et al., 2016).

Em oposição a afirmativa, Jam et al. (2018) em seu estudo realizado com 97 enfermeiros que atuavam em UTI's de hospitais da Espanha, afirma que, cargas de trabalho e grau de conhecimento não influenciaram a adesão dos profissionais a medidas de prevenção. Onde o estudo poderia ser base para instigar novas investigações, pois não chegou a resultados definitivos. Neste ponto, Shahnaz et al. (2018) afirmam que a adesão dos profissionais também tem relação com o fato de o hospital ser público ou privado, o que poderia ser explicado pela existência de maior padronização dentro do setor privado.

O estudo de Aysegul, Oznur e Asiye (2020), afirmam que, os profissionais têm conhecimento e utilizam a prática baseada em evidências para orientar suas ações, foi observado que os profissionais seguem protocolos de medidas preventivas e aderem a novas medidas que tenham comprovação científica. Porém, os autores destacaram que os profissionais se descuidam da própria saúde, onde dos 102 enfermeiros entrevistados, 57,8\% não haviam tomado vacina contra gripe, o que poderia aumentar as chances de os pacientes em uso de ventilação mecânica adquirir pneumonia.

Merece destaque por colaborar fortemente para a não adesão as medidas de prevenção da pneumonia associada à ventilação mecânica em UTI, a falta de educação continuada, falta de padronização, e altas cargas de trabalho (Aloush et al., 2018). Onde Branco et al. (2020) afirmam que a prática de educação continuada aumenta a adesão a medidas de prevenção da pneumonia associada a ventilação mecânica.

Deve-se destacar ainda a necessidade da realização de treinamentos e educação continuada para que os profissionais possam aderir e dar continuidade ao uso das medidas de prevenção (Aloush, 2018). Outras barreiras a adesão de medidas de prevenção que devem ser citadas são: a escassez de profissionais e as políticas de gastos de cada instituição, que podem dificultar determinadas ações (Al-Sayaghi, 2021).

\section{Considerações Finais}

Este estudo permitiu descrever os cuidados preventivos de enfermagem acerca da pneumonia associada a ventilação mecânica. Onde se destacaram os principais fatores de risco como: alterações nos umidificadores e nos circuitos, não realização da higiene oral, não adesão às medidas preventivas e outras. Foram apresentados ainda, os fatores que influenciam a adesão de medidas de prevenção da pneumonia associada a ventilação mecânica e bundles de intervenções.

Diante dos problemas encontrados, torna-se claro que a assistência prestada deve ser focada tanto no processo de adoecimento que o paciente vivencia quanto na prevenção de outras patologias, como é o caso da pneumonia, que pode gerar diversas complicações e até mesmo levar ao óbito.

Os enfermeiros exercem importante papel dentro da UTI e são importantes colaboradores para a prevenção de pneumonia associada à ventilação mecânica, assim, torna-se necessário realizar treinamentos e fornecer educação continuada para esses profissionais, a fim de provocar uma sensibilização sobre o uso de medidas preventivas e entender todos os fatores que influenciam a adesão dos enfermeiros. 
Research, Society and Development, v. 11, n. 3, e19611326022, 2022

(CC BY 4.0) | ISSN 2525-3409 | DOI: http://dx.doi.org/10.33448/rsd-v11i3.26022

Como limitações do estudo tem-se o fato de que, a maioria das pesquisas não conta com a participação direta dos pacientes, pois os mesmos, muitas vezes, não podem se comunicar. Além disso, destaca-se que a literatura oferece muitos estudos, porém, observou-se que alguns aspectos ligados a pneumonia associada à ventilação mecânica são mais investigados que outros, o que poderia interferir nos resultados da pesquisa.

Por fim, o estudo possibilitou expandir os conhecimentos acerca da UTI, da atuação do enfermeiro e da pneumonia associada à ventilação mecânica. Dessa forma, considera-se que novas pesquisas devem ser realizadas nesta linha de investigação, a fim de comprovar os benefícios das medidas preventivas e estimular o seu uso nas unidades de terapia intensiva.

\section{Referências}

Alcan, A. O., Korkmaz, F. D., \& Uyar, M. (2016). Prevention of ventilator-associated pneumonia: Use of the care bundle approach. Am J Infect Control, 44(10), 173-176.

Almeida, K. M. V. D., Barros, O. M. C. D., Santos, G. J. D., Valença, M. P., Cavalcanti, A. T. D. A., \& Ferreira, K. O. (2015). Adesão às medidas de prevenção para pneumonia associada à ventilação mecânica. Revista de Enfermagem da Ufsm, 5(2), 247-256.

Aloush, S. M. Abdelkader, F. A., Al-Sayaghi, K., Tawalbeh, L. I., Suliman, M., Bashtawy, M. A., \& Insaf Shaban. (2018). Compliance of nurses and hospitals with ventilator associated pneumonia prevention guidelines: a middle eastern survey. J Nurs Care Qual., 33(3), 1-7.

Aloush, S. M. (2018). Nurses' implementation of ventilator-associated pneumonia prevention guidelines: an observational study in Jordan. British Association of Critical Care Nurses, 23(3), 147-151.

Al-Sayaghi, K. M. (2021). Critical care nurses' compliance and barriers toward ventilator-associated pneumonia prevention guidelines: cross-sectional survey. Journal of Taibah University Medical Sciences, 16(2), 274- 282.

Aysegul, C., Oznur, U. Y., \& Asiye, A. (2020). Evidence-Based Practices for Preventing Ventilator-Associated Pneumonia in Intensive Care Nursing: Knowledge and Practice. International Journal of Caring, 13(3), 1794.

Branco, A., Lourençone, E. M. S., Monteiro, A. B., Fonseca, J. P., Blantt, C. R., \& Caregnato, R. C. A. (2020). Educação para prevenção da pneumonia associada à ventilação mecânica em unidade de terapia intensiva. Rev. Bras. Enferm, 73 (6).

Darawad, M. W., Sa'Aleek, M. A., \& Shawashi, T. (2018). Evidence-based guidelines for prevention of ventilator-associated pneumonia: Evaluation of intensive care unit nurses' adherence. American Journal of Infection Control, 46(6), 711-713.

Fan, Y., Chu, X., Jiang, L., \& Du, x. (2021). The clinical value of comprehensive nursing intervention in preventing ventilator-associated pneumonia. American Journal of Translational Research, 13(4), 3845-3850.

Gonçalves, A. F., Brasil, V. V., Ribeiro, L. C. M., \& Tipple, A. F. V. (2012). Ações de enfermagem na profilaxia da pneumonia associada à ventilação mecânica. Acta Paulista de Enfermagem, 25(1), 101-107.

Hassan, Z. M., \& Wahsheh, M. A. (2017). Knowledge level of nurses in Jordan on ventilator-associated pneumonia and preventive measures. British Association of Critical Care Nurses, 22(3), 125-132.

JAM, R., Mesquida, J., Hernández, O., Sandalinas, I., Turégano, C., Carrillo, E., ...\& Delgado-Hito, P. (2018). Nursing workload and compliance with nonpharmacological measures to prevent ventilator-associated pneumonia: a multicentre study. British Association of Critical Care Nurses, 23(6), $291-298$.

Alves, D. L., Oliveira, F. B. M. (2016). Relação entre a sobrecarga de trabalho e erros de administração de medicação na assistência hospitalar. Revista Ciências \& Saberes, 2(2).

Ribeiro, K. R. A., Anjos, E. G., \& Oliveira, E. M. (2016). Enfermagem em ventilação mecânica: cuidados na prevenção de pneumonia. Revista Recien., 6(16), 57-71.

Severino, A. J. (2017). Metodologia do trabalho científico. São Paulo: Cortez.

Shahnaz, A., Bhadwaj, U., \& Tamag, K. (2018). A Comparative Study to Assess the Competency among Icu Nurses in Using Ventilator Associated Pneumonia Bundle to Prevent Ventilator Associated Pneumonia in Selected Government and Private Hospitals of New Delhi. International Journal of Nursing Education, 10(3).

Silva, M. C. O., \& Moura, R. C. M. (2016). Cuidados de enfermagem na prevenção da pneumonia associada à ventilação mecânica: revisão integrativa. Carpe Diem: Revista Cultural e Científica do UNIFACEX, 14(2), 74-85.

Souza, M. T., Silva, M. D., \& Carvalho, R. (2010). Revisão integrativa: o que é e como fazer. Einstein, 8, 102-6.

Zigart, J. A. A., Contrin,L.M., Frutuoso, I. S., Silveira, A. M. R. D., Beccaria,L.M., \& Alexandre Lins Werneck, A. L. (2019). Adesão ao protocolo de pneumonia associado à ventilação mecânica. Rev enferm UFPE on line., 13(1), 655-663. 